\title{
Study on Antioxidant Activity of Alcohol Extracts from Persimmon
}

\author{
Huan Chen ${ }^{1}$, Zhaobiao Luo ${ }^{2}$ \\ 1 Nanchang Institute of Science \& Technology, Nanchang City, Jiangxi \\ Province, China, 330108 \\ 2 Fuzhou Tobacco Company in Jiangxi Province,Fuzhou City, Jiangxi \\ Province, China, 344000
}

\begin{abstract}
In the body due to some exogenous factors and endogenous metabolic processes, leading to oxygen molecules produce reactive oxygen species. In the normal metabolic process, reactive oxygen intervention is harmful, reactive oxygen species within and outside the cell to initiate a variety of biologically active compounds interact, eventually leading to the body to produce pathological changes. In recent years, many natural antioxidants and synthetic antioxidants have been developed and studied in order to protect biological macromolecules from active oxygen attack and inhibit damage to living bodies caused by reactive oxygen species. Synthetic antioxidants, such as dibutyl light toluene (BHT) and dibutyl light light aromatic ether (BHA), have high antioxidant activity. However, the synthesis of antioxidants has potential hazards, therefore, in the field of food chemistry development of safe and effective natural antioxidant has become an important research hotspot
\end{abstract}

Keywords: antioxidant activity, alcohol extracts, persimmon

\section{Introduction}

Persimmon in China there are vast resources and development and utilization of space. Food processing organizations are now a variety of development and utilization of persimmon flesh. Anti-aging, anti-microbial, hemostasis, and has 
entered the clinical application, has broad application value. Therefore, it has been widely used in the treatment of cardiovascular diseases, anti-tumor, antiaging, anti-microbial and hemostasis. Persimmon fruit is generally believed that the antioxidant effect is mainly due to its pulp containing polyphenols. Also reported dry persimmon skin antioxidant, can lower blood cholesterol, with antiatherosclerotic effect. Persimmon seeds contain tannin compounds, has a strong antioxidant activity, can effectively remove the liver of peroxide reactants and reduce the level of hydrogen peroxide lecithin. However, the antioxidant activity of persimmon and its alcohol extract in vitro has not been systematically studied. Persimmon is a low-fat and rich rich antioxidant activity of food, its ethanol or methanol extract can effectively prevent atherosclerosis. The flesh of the dietary fiber, minerals, trace elements and polyphenols have significant antioxidant activity. Diet-induced hyperglycemia in rat blood serum showed that persimmon could improve blood lipid metabolism and decrease lipid. It has also been reported that persimmon leaves contain a large number of tannin substances, can prevent the treatment of hypertension. Rat experiments show that isolated from the persimmon flavonoid substances can play a role in lowering blood pressure. Persimmon polyphenols including persimmon tannin together play a variety of different physiological effects. Persimmon leaf tea has a good antioxidant properties, in the water extract of persimmon leaf tea and methanol extract of the antioxidant test showed that the water extract in carrot / linoleic acid system of total antioxidant capacity detection is extremely strong Antioxidant activity, $0.125 \%$ higher than $10 \mathrm{mM}$ vitamin c. For the removal of 0 larvae, the methanol extract was found to be more potent than the aqueous extract. Both extracts had a strong ability to scavenge DPPH., And $0.1 \%$ of the water extract inhibited DPPH more than $90 \%$, whereas $1 \%$ of the extract was comparable to $1 \mathrm{mM}$ vitamin $\mathrm{C}$ clearance $\mathrm{OH}$. It has been reported that long-term drinking persimmon leaves can play a role in the treatment of chronic diseases.

\section{Materials and methods}

(DPPH), phenazinemethosulphate (PMS), linoleic acid (gallic acid), gallic acid (PMS) were prepared by the reaction of $\beta$-carotene with Merck, L-ascorbic acid, $\mathrm{K} 3 \mathrm{Fe}(\mathrm{CN})$ 6,2-deoxy-D-ribose (DR), nitrogen blue tetrazolium (NBT), NADH, 2- Thiobarbituric acid (TBA) are made of analytical grade. 1.3 Preparation of EP and determination of related components.

And then the mixture was homogenized with 1:10 (persimmon / 70\% alcohol, $\mathrm{W} / \mathrm{V}$ ), refluxed at $80{ }^{\circ} \mathrm{C}$ for $3 \mathrm{~h}$, and the crude extract was filtered with gauze. The floc was filtered under reduced pressure and evaporated in vacuo at $50^{\circ} \mathrm{C}$ and concentrated. The concentrated extract was dried in an oven at $50^{\circ} \mathrm{C}$ to obtain an extract. Store at $4{ }^{\circ} \mathrm{C}$ in the refrigerator.

The concentration of gallic acid $(0.1 \mathrm{mg} / \mathrm{ml}$ methanol) was used as the standard, and the absorbance was measured at $770 \mathrm{~nm}$ using the Folin reagent method. The content of total polyphenols was calculated by linear regression 
equation. The concentration of total flavonoids was determined by spectrophotometry with rutin $(0.2 \mathrm{mg} / \mathrm{ml}, 60 \%$ ethanol) as the standard, absorbance at $510 \mathrm{~nm}$ and linear regression equation.

The phenol content was measured by phenol-sulfuric acid method, and glucose was taken as the standard, and the linear regression equation was used to calculate the glucose content. Extract extract extract 2, a copy of distilled water dissolved, for the determination of total sugar content. Add another distilled water, add 3 times the volume of distilled water, ethanol mixture, static $2 \mathrm{~h}, 4000 \mathrm{r}$ / min centrifugation $10 \mathrm{~min}$. The precipitate was washed twice with anhydrous ethanol, acetone and ether respectively, dried in a water bath at $50{ }^{\circ} \mathrm{C}$ and dried in an oven at $105{ }^{\circ} \mathrm{C}$ for 12 hours to obtain constant weight polysaccharide. Weigh the weight of polysaccharide powder prepared into the final working solution $0.2 \mathrm{mg} / \mathrm{ml}$, for the determination of polysaccharide content.

According to Yamaguchi et al proposed DPPH free radical analysis method, measured at $517 \mathrm{~nm}$ absorbance. Inhibition rate $(\%)=(1-\mathrm{Ae} / \mathrm{Ao}) 100$ where A o is the absorbance of the unadded sample; A e is the absorbance of the sample.

(NBT) reduction method was used to determine the activity of EP on superoxide anion scavenging ability, measured at 560nm absorbance, VC as a positive control. Suppression rate $(\%)=(1-\mathrm{E} / \mathrm{C}) \times 100$ where $\mathrm{E}$ is the absorbance of the sample group, and the absorbance of the sample control group is the absorbance of the sample control group. In order to eliminate the effect of the sample on the experimental results ; $\mathrm{C}$ is the absorbance of the sample is not added. The scavenging activity of crude extracts on hydroxyl radicals was determined by 2-deoxy-D-ribose method. The absorbance was measured at 532 $\mathrm{nm}$, and D-mannitol was used as a positive control. In order to eliminate the influence of the sample on the experimental results, the concentration of each sample were set to control the inhibition rate calculated. Total antioxidant activity was determined from peroxides generated from the autoxidation system of [beta] -carotene / linoleic acid. The measured absorbance at 470nm, positive control for the $\mathrm{V} \mathrm{C}, \mathrm{B} \mathrm{H} \mathrm{T}$, absorbance, said its strong antioxidant activity. The reducing power of EP was determined by the reduction ferric ion method proposed by Oyaizu M et al. At 700nm measured absorbance, VC, BHT as a positive control, a large absorption of its ability to restore strong.

Lipid peroxidation was induced by $\mathrm{Fe} 2+$ and lipid peroxidation was evaluated by measuring the content of malondialdehyde in the final product. At $532 \mathrm{~nm}$ measured absorbance values, $\mathrm{VC}$ as a positive control. In order to eliminate the influence of the sample on the experimental results, the concentration of each sample were set to control the inhibition rate calculated with.

\section{Results and analysis}

$26.69 \mathrm{~g}$ of the extract-like extract was recovered in a yield of $13.35 \%$. Also called $1.063 \mathrm{~g}$ extract $\mathrm{EP}$, at $105{ }^{\circ} \mathrm{C}$ drying $12 \mathrm{~h}$, was constant weight $0.9614 \mathrm{~g}$, was extract in the dry matter content of $90.44 \%$. According to the linear regression 
equation $\mathrm{y}=0.06 \mathrm{x}-0.0133(\mathrm{R} 2=0.948$, where, $\mathrm{y}$ is the concentration, $\mathrm{x}$ is the absorbance), the total content of polyphenols in extract EP is $0.33 \%$.

( $\mathrm{R} 2=0.9989$, where $\mathrm{A}$ is the absorbance and $\mathrm{x}$ is the concentration), and the content of monosaccharide in EP was determined as follows: $\mathrm{A}=6.68 \mathrm{x}-0.0422$ ( $\mathrm{R} 2=0.9989$, where $\mathrm{A}$ is the absorbance and $\mathrm{x}$ is the concentration) $29.49 \%$. According to the conversion coefficient of oligosaccharide to monosaccharide 0.9 and the polysaccharide content $32.54 \%$, the total sugar content was $62.03 \%$.

There are literatures that the alcohol extract of persimmon is stronger than the water extract in scavenging DPPH, which may be related to the polyphenols and other active ingredients soluble in alcohol solvents. As can be seen from Table 1, EP on DPPH free radical scavenging effect, the IC50 of $0.8 \mathrm{mg} / \mathrm{ml}$, and a significant dose-dependent. However, the IC50 of synthetic BHT was $0.037 \mathrm{mg} /$ $\mathrm{ml}$. VC had higher scavenging ability to DPPH than BHT and EP. Therefore, it was necessary to increase the dosage of EP instead of V C and B H T. However, $\mathrm{E} P$ is a natural food extract, in terms of safety has obvious advantages.

E P can effectively remove $\mathrm{O} 2$ generated in vitro, the IC50 is $2.1 \mathrm{mg} / \mathrm{ml}$. Although its ability to remove $\mathrm{O} 2 \cdot$ than $\mathrm{VC}$ (IC50 of $0.052 \mathrm{mg} / \mathrm{ml}$ ) is weak, but in the same natural extract, IC50 can reach $2.1 \mathrm{mg} / \mathrm{ml}$, is already very valuable, and the stability of EP than VC Much better. Therefore, the role of E P in the removal of $\mathrm{O} 2 \cdot$ is worthy of exploitation.

B - carotene is easily oxidized to fade yellow. In the reaction system, the oxidation of linoleic acid produces peroxide to discolor $\beta$ - carotene. When the reaction system contains antioxidant, the fading rate is slow, and the degree of fading and antioxidant Activity was negatively correlated. As shown in Table 4, E P, B H T and V C all showed strong total antioxidant activity, and the activity was stable. The antioxidant capacity was in the order of BHT $>$ VC $>$ EP. Although the total antioxidant capacity of EP is slightly lower than that of VC and BHT, the stability of EP is stronger than that of $\mathrm{VC}$, and the safety is better than that of BH. In conclusion, EP has a good antioxidant effect.

Lipid in the reactive oxygen species under free radicals, lead to chain reaction, the formation of lipid peroxidation. Antioxidants with lipid peroxidation of lipid free radicals or lipid free radical reaction, the termination of chain reaction, thus inhibiting lipid oxidation. Low density lipoprotein oxidation is one of the pathogenesis of atherosclerosis, and thus free radical scavenging, anti-lipid peroxidation is the prevention and treatment of atherosclerosis important process of protection measures. It can be seen from Table 6, E P on lipid peroxidation better inhibition, and a better dose-dependent, the IC50 of $4.2 \mathrm{mg} / \mathrm{ml}$.

Free radicals produced by the metabolism of the body, is a by-product of metabolic processes, including light-based free radicals, superoxide anion radicals, peroxides, etc., they can cause damage to the body. Under normal circumstances, the body there is a perfect free radical scavenging system, so that the production and elimination of free radicals in the dynamic equilibrium. Under stress, free radicals produced by the body can not be removed in time, and the active free radicals and the unsaturated fatty acids in the free or bound state not only change the structure and function of the membrane, but also induce the increase of lipid peroxidation products. In recent years, many studies have shown 
that lipid peroxidation induced by oxygen free radicals is closely related to the occurrence of many diseases, such as physical factors, biological factors, immune injury leading to various inflammation of the body, atherosclerosis, cerebral artery spasm, Senile dementia, aging, etc., and exogenous free radical scavengers can reduce the level of free radicals in the body, so that the body to maintain a good condition. Therefore, domestic and foreign scholars are actively engaged in screening with oxygen scavenging free radicals and anti-lipid peroxidation of effective drugs.

\section{Conclusion}

EP has a strong ability to scavenge DPPH. It can effectively scavenge free radicals. It has a strong scavenging effect on O-butyrolactone. It has obvious inhibition on lipid peroxidation. It has strong total reducing power and total antioxidant activity. Oxidative power, and have a good dose-dependency. EP can effectively protect mitochondria from oxidative damage induced by eZi2 or mitochondria, remove mitochondrial MDA, slow vc / $\mathrm{Fe} 2+$-induced mitochondrial swelling, protect mitochondrial ATPase activity, and have a strong effect on protein-based levels Inhibition.

\section{References}

[1] Wang Tongyang. Persimmon Acetic Acid Beverage Fermentation Process Research. China Brewing. 55(12), pp.68-70, 2011

[2] Chen Zhenlin, Yang Huiling, Huang Zhiqiang, Zhang Zhi. Brewing and Nutritional Components Of Persimmon Fermented Wine. Food Science Bulletin 8 (5), pp.87- 91, 2013

[3] Yuan Jiangfeng, Yang Jianxiong, Zhang Zhiqi, Huang Yaya.Effects of Producing Area and Season on the Content of Total Flavonoids, Rutin And Oleanolic Acid In Persimmon Leaves. Chinese Traditional And Pharmacy. 12(10), pp. 18- 20, 2012

[4] Chen Xiangning, Wang Wazhuang, Wu Xuerui, Kong Xiaojiao, Yue Xi, Li Lite. Study On The Relationship Between Different Components And Antioxidant Activity Of Persimmon. Food Science, , 9(6), pp.58- 61, 2011

[5] Zhang Yali, Mei Jiansheng, Zhang Meiyue. Extraction and Stability of Persimmon Pigment. Food Science \& Technology. 8(4), pp.85-87, 2014 